\title{
Validation of digital holographic tomography in flow measurement
}

\author{
Pavel Psota ${ }^{1 *}$, Roman Doleček ${ }^{1}$, Vit Lédl ${ }^{1}$, Tomáš Vít ${ }^{2}$, Pavel Mokrý1 ${ }^{1}$, and Petra Dančová2 \\ ${ }^{1}$ Regional Centre for Special Optics and Optoelectronic Systems (TOPTEC), Institute of Plasma Physics, Academy of Sciences of the \\ Czech Republic, Za Slovankou 1782/3, 18200 Prague 8, Czech Republic \\ ${ }^{2}$ Institute of Energetic Devices (KEZ), Faculty of Mechanical Engineering, Technical University of Liberec, Studentská 2, 46117 \\ Liberec, Czech Republic
}

\begin{abstract}
This paper reports on measurements by digital holographic tomography in flow measurement. It is demonstrated capability of the method by measurement of general temperature field consisting of system of three orifices. Results obtained by digital holographic tomography are compared to results obtained by hot wire anemometry CTA.
\end{abstract}

\section{Introduction}

Digital holographic interferometry (DHI) for measurement and visualization of temperature fields is well established tool in the field of the fluid mechanics [1-4]. DHI is very sensitive to faint temperature variations that are measured by a laser beam propagating through the inspected area and thus the method is noninvasive. However, due to integral nature of interferometric techniques, for general temperature fields the conventional DHI must be replaced by more complex method called digital holographic tomography (DHT) [1,5]. DHT provides information of the temperature variations within the whole volume with great lateral and temperature resolution. Since DHT is quite new approach in the fluid engineering the authors want to lay the DHT on the firm foundation by comparison of DHT to generally accepted technique like Constant Temperature Anemometry (CTA) [6]. This paper briefly introduces basic principles of DHT, shows some possible application in fluidics and the results are verified by CTA.

\section{Digital holographic tomography in fluid mechanics}

\subsection{Digital Holography}

Digital holography comprises recording of the digital hologram and its reconstruction. For recording, CCD or CMOS sensors are used to capture microinterference pattern (digital hologram) in which the information about the phase of the incoming waves is encoded. The hologram $H$ is formed by a superposition of a reference wave $U_{r}$ and an object wave $U_{o}$ :

$$
H \approx\left|U_{o}+U_{r}\right|^{2}=\left|U_{o}\right|^{2}+\left|U_{r}\right|^{2}+U_{o} U_{r}^{*}+U_{r} U_{o}^{*} .
$$

Digitally recorded hologram is transferred to a computer as an array of numbers that represent the wave field at the hologram plane. Reconstruction of the digital hologram is done numerically by a computer. The propagation of optical fields is completely described by diffraction theory, which allows numerical reconstruction of the image as an array of complex numbers representing the amplitude and phase of the optical field. The free space propagation can be solved by the convolution theorem [1]

$$
\begin{aligned}
& U(x, y)= \\
& \quad=\mathfrak{I}^{-1}\left\{\mathfrak{I}\left(H(\xi, \eta) r^{*}(\xi, \eta)\right) \mathfrak{I}(g(x, y))\right\}
\end{aligned}
$$

with the impulse response:

$$
g(x, y)=\frac{1}{j \lambda} \frac{\exp \left(j k \sqrt{d^{2}+x^{2}+y^{2}}\right)}{\sqrt{d^{2}+x^{2}+y^{2}}} .
$$

The Fourier transform of $g(x, y)$ can be calculated and expressed analytically as well. This saves one Fourier transform for reconstruction.

\subsection{Temperature fields measurement by DHT}

It is well know that a temperature variation in fluids is accompanied by a variation of refractive index of the fluid. This feature can be exploited for measurement of the temperature through the knowledge of the refractive index change. When two waves propagate through a medium with certain refractive indexes their phase difference $\Delta \varphi$ varies with respect to the change of the 
refractive indexes $\Delta n$. This is mathematically expressed by formula

$$
\Delta \varphi=\frac{2 \pi}{\lambda} \oint_{L} \Delta n d l
$$

where $d l$ denotes the differential distance along the line $L$ and $\lambda$ is the wavelength of the wave. Therefore, to retrieve information about the refractive index change one has to solve (4).

The solution is quite straightforward for two dimensional temperature fields (integral is simplified to multiplication) and for symmetrical temperature fields (leads to Abel transform), however, for general distribution of temperature fields the solution of (4) without any additional information is impossible. Therefore it is required to use tomographical approach.

The refractive index change in (4) can be written in polar coordinates $(t, \Phi)$ as

$$
\Delta n(y, z)=\Delta n(t \cos \Phi, t \sin \Phi) .
$$

The determination of general fields requires the analysis of a large number of phase projections by methods of tomography. In this case the line integral transform (1) is mathematically equivalent to Radon Transform:

$$
\Delta \varphi(s, \theta)=\iint \frac{2 \pi}{\lambda} \Delta n(y, z) \delta(y \cos \theta+z \sin \theta-s) d y d z,
$$

where $\Delta n(y, z)$ is the refractive index distribution in Cartesian coordinates, $\delta$ is the Dirac pulse, $\theta$ is the angle of projection and $\mathrm{s}$ is the shift distance from the origin. The reconstruction of the refractive index change is based on back-projection mathematically described by Inverse Radon Transform [1]:

$$
\Delta n(y, z)=\frac{\lambda}{2 \pi} \int_{0}^{\pi} g(y \cos \theta+z \sin \theta, \theta) d \theta .
$$

The back-projection operation propagates the measured phase information back into the image space along the projection paths in order to get $3 \mathrm{D}$ volume data of the refractive index change.

The phase at one projection can be measured by digital holographic interferometry where two digital holograms $H_{1}, H_{2}$ are captured by a digital sensor. The first hologram corresponds to an initial (reference) state of a temperature field and the second hologram is captured when the temperature field has been varied. Two wave fields are reconstructed from the digital holograms as complex fields represented by formula:

$$
\begin{aligned}
U_{1}(n \Delta x, m \Delta y)= & U_{1}(n \Delta x, m \Delta y) \mid \times \\
& \times \exp \left[\varphi_{1}(n \Delta x, m \Delta y)\right], \\
U_{2}(n \Delta x, m \Delta y)= & \left|U_{2}(n \Delta x, m \Delta y)\right| \times \\
& \times \exp \left[\varphi_{2}(n \Delta x, m \Delta y)\right],
\end{aligned}
$$

respectively. When the wave fields are divided one by another $U_{1} / U_{2}$, their phase difference $\Delta \varphi$ is subtracted, and it can be shown that for the phase difference $\Delta \varphi$ holds an expression:

$$
\Delta \varphi=\arg \left(\frac{\operatorname{Im}\left\{U_{1}\right\} \operatorname{Re}\left\{U_{2}\right\}-\operatorname{Im}\left\{U_{2}\right\} \operatorname{Re}\left\{U_{1}\right\}}{\operatorname{Im}\left\{U_{1}\right\} \operatorname{Im}\left\{U_{2}\right\}+\operatorname{Re}\left\{U_{1}\right\} \operatorname{Re}\left\{U_{2}\right\}}\right) .
$$

\section{Experimental arrangements}

The DHT measurement was realized by TwymannGreen interferometer, see Fig. 1. The spatially filtered (SF) and collimated (CO) laser beam is split by polarizing beam splitter BS1. The reference beam (RW) goes through mirrors M1, M2 and beam splitter BS2 to the digital camera (CAM). The object beam (OW) goes twice (before and after reflection at M3) through the measured area (SAMPLE) and after the beam splitter BS2 impinges the CAM where the both beams are superposed creating the digital hologram.

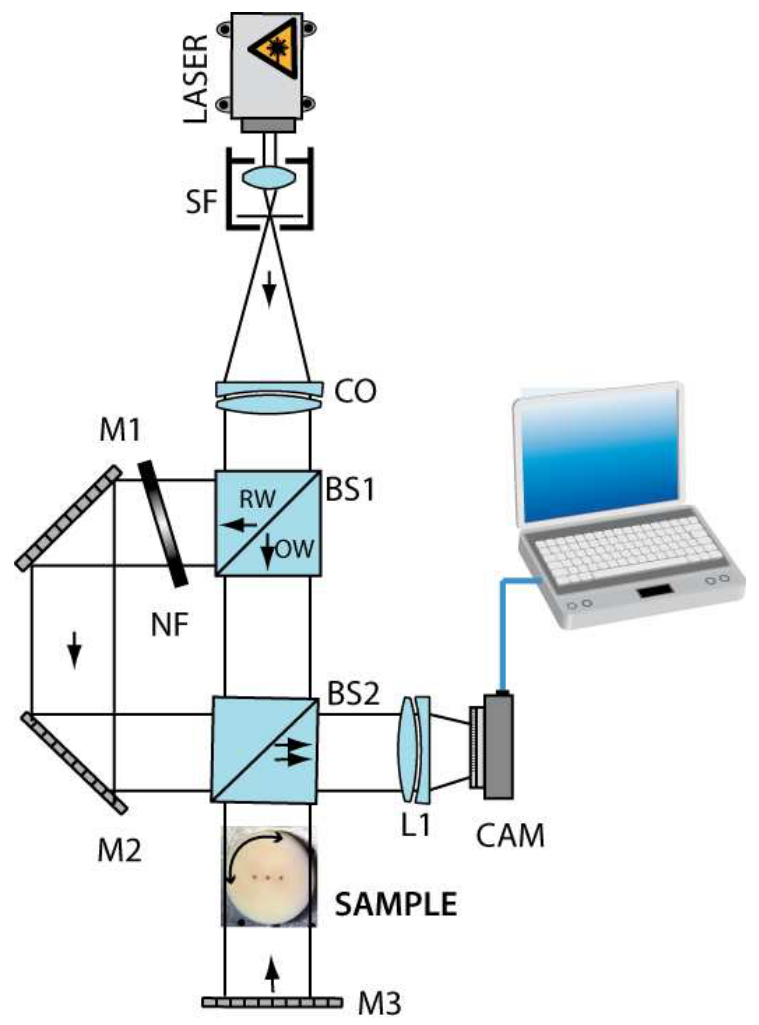

Fig. 1. Experimental holographic arrangements schematics of interferometer with double pass for tomographic measurement. BS1-polarizing beam splitter, SF-spatial filter, CO-collimation objective, BS2-nonpolarizing beam splitter, L1-objective lens, M-mirror, NF-neutral filter.

This setup forces the beam to pass through the measured object twice which results in higher sensitivity $[3,5]$.

To demonstrate the reliability of the method we measured a non-symmetrical temperature field of heated air flowing out from a three orifices system. The outgoing air was heated inside the cavity under the orifice by a heating cartridge in order to increase its temperature difference to surrounded fluid. The 
temperature of heating cartridge was set to $100^{\circ} \mathrm{C}$ and regulated by $\mathrm{PID}$ regulator.

The measured object was placed on the $360^{\circ}$ rotation stage and rotated step by step at angle of $10^{\circ}$ from $0^{\circ}$ to $170^{\circ}$ to get projections from different viewing angles. At every step a sequence of digital holograms is captured and reconstructed. The reconstructed phase fields at the same projection angle were averaged out in order to suppress the random phenomena. This measurement procedure was repeated for every angle step. Before the measurement we captured a sequence of reference digital holograms when the heated system was off.

The representation of the reconstructed phase data as a function of the angle is known as a sinogram. The 3-D temperature distribution can be reconstructed from the sinograms by a filtered backprojection algorithm, see (7). For this purpose, the standard inverse Radon transform (iradon command) in MATLAB was used with Ram-Lak filter and implemented slice-by-slice along the rotation axis. Reconstructed temperature distribution in 3D space is shown in Fig. 2.

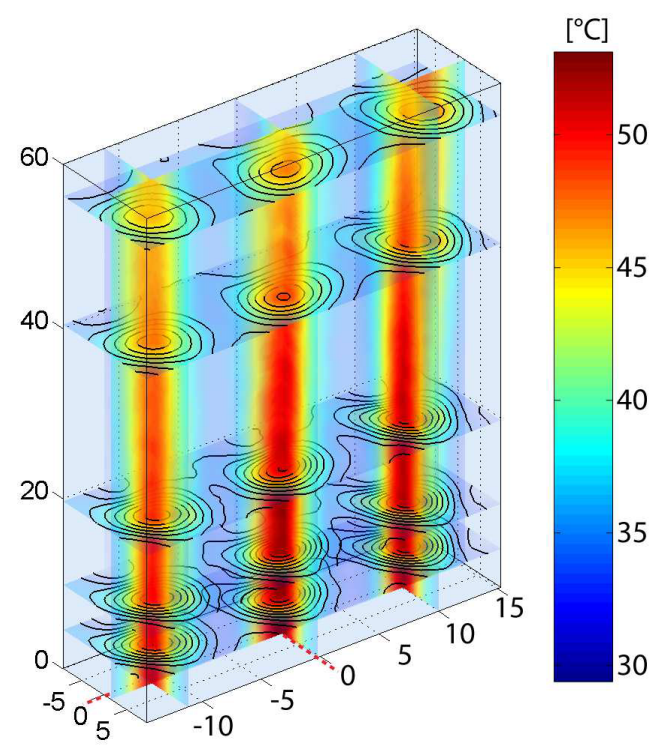

Fig. 2. 3D temperature distribution measured by DHT. Axes units are $\mathrm{mm}$

\section{Results and Discussions}
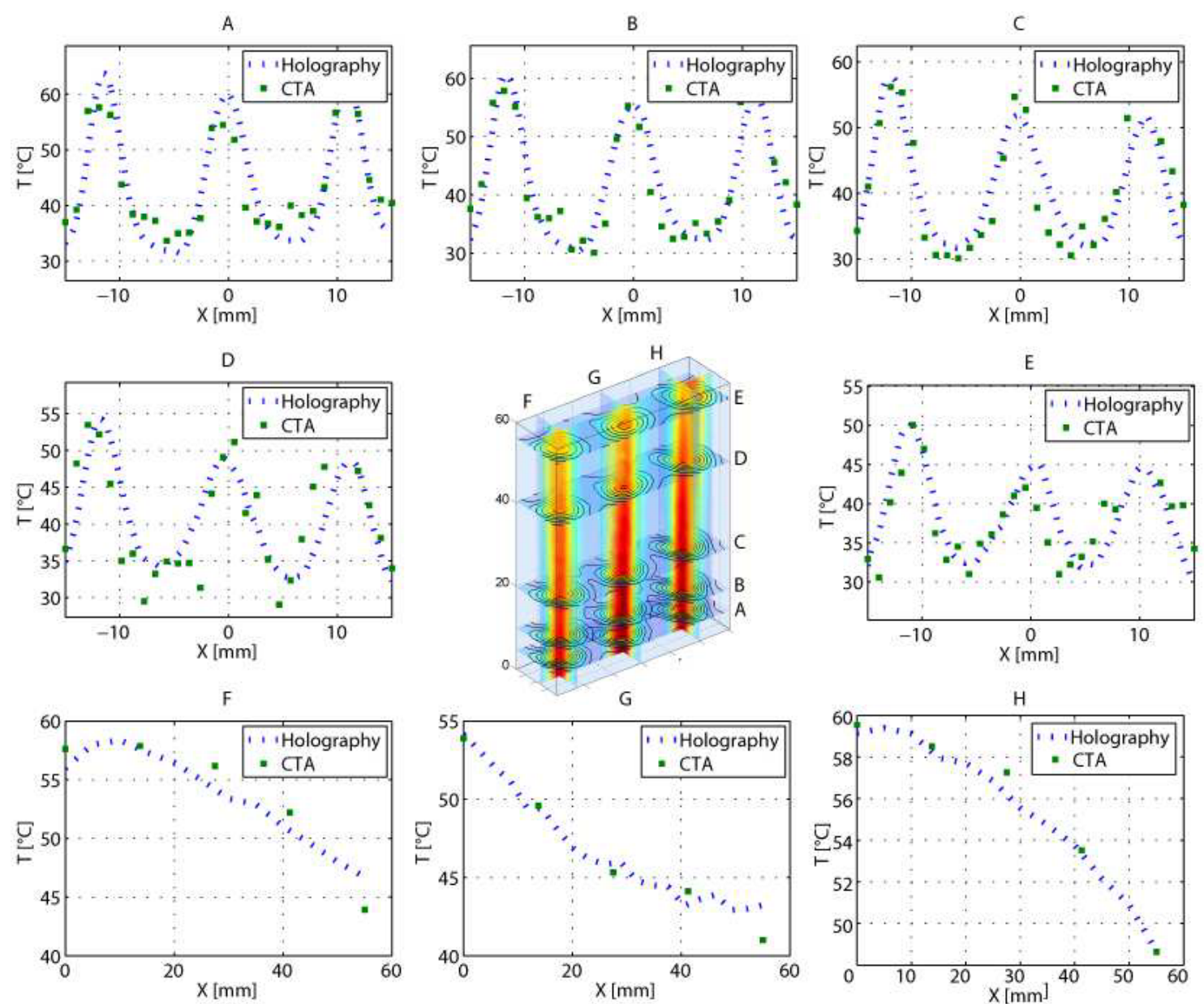

Fig. 3. Comparison of DHT and CTA along different slices as illustrated in the $3 \mathrm{D}$ image.

To verify the reliability of digital holographic tomography we compared results of DHT with measurement results of well-established CTA (Constatnt Temperature Anemometry). CTA is single point method and therefore we chose some representative points 
within the measured area. The CTA measurements were carried out along horizontal lines at different heights. The results at different heights $(5 \mathrm{~mm}, 10 \mathrm{~mm}, 20 \mathrm{~mm}$, $40 \mathrm{~mm}$ and $55 \mathrm{~mm}$ ) are denoted by the letters A-E in Fig. 3. The distance of measuring step by CTA in horizontal direction was $1 \mathrm{~mm}$. DHT is a full-field method and therefore we had to pick voxels of the measured temperature distribution corresponding to those values measured by CTA.

Results are presented in Fig. 3. 1D plots denoted A-E present results obtained by DHT and CTA along horizontal lines with the same label. The graphs denoted by letters F-H shows how the temperature varies along vertical line (axis of the orrifices).

To quantitatively determine the difference of the method we computed RMS difference $\Delta T_{R M S}$ at all measured points $p$ :

$$
\Delta T_{R M S}=\sqrt{\frac{\sum_{p=1}^{N}\left(T_{D H T}(p)-T_{C T A}(p)\right)^{2}}{N}}=3.8^{\circ} \mathrm{C}
$$

The RMS (root-mean-square) difference is $3.8{ }^{\circ} \mathrm{C}$ that can be expressed as an agreement in the range of cca $12 \%$.

It is worthwile mentioning the main sources of the disrepancies in results between DHT and CTA. The major contributor is fact that the both measurements had to be performed separately at different time due to invasive feature of CTA. The enviromental conditions as well as behaviour of the phenomenon itself had been probably slightly varying between both meausurement procedures.

The uncertainty of measurement by DHT is also affected by the number of projection used in inverse Radon transform. The angle step between succesive projections was set to be $10^{\circ}$. This generates an error of cca $0.4{ }^{\circ} \mathrm{C}$ for temperature values typical for our system, see Fig. 4.
A

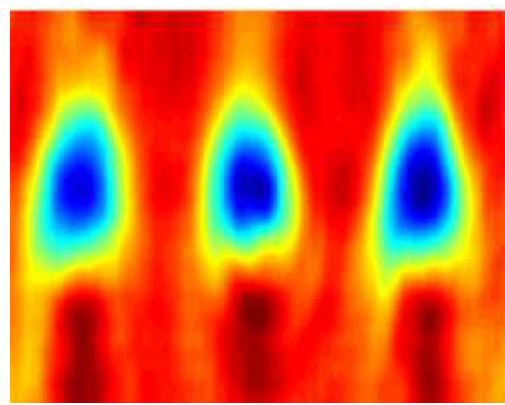

B

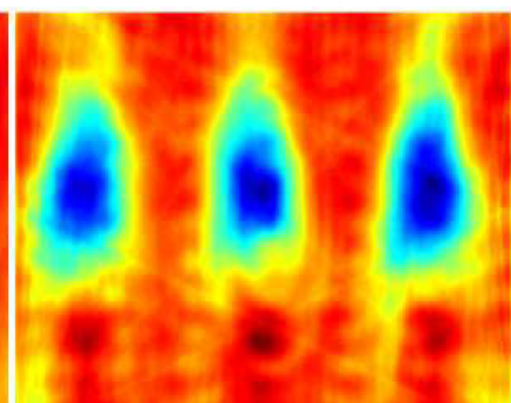

C

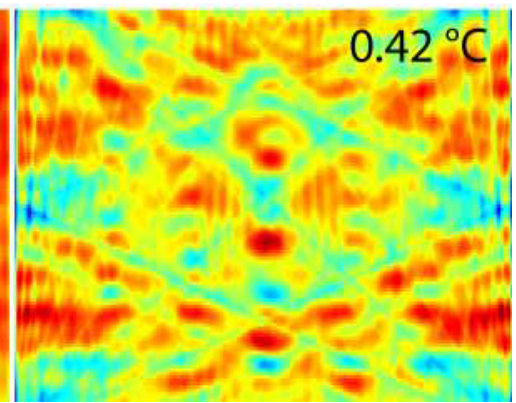

Fig. 4. Simulation of effect of low angle sampling in tomography. A) original (reference) image. B) image obtained by inverse Radon transform with step of $10^{\circ}$. C) RMS difference (error) between A and B. The theoretical error introduced by low angle sampling in our experiments is $0.42^{\circ} \mathrm{C}$.

We gratefully acknowledge the support of the Grant Agency of the Czech Republic - Czech Science Foundation (projects no. 14-08888S and 14-32228S).

\section{References}

1. T. Kreis, Handbook of holographic interferometry: optical and digital methods. 2005.

2. C. Shakher and A. K. Nirala, Applied optics, 33, 11, (1994).
3. V. Lédl, T. Vít, R. Doleček, and P. Psota, EPJ Web of Conferences, 25, (2012)

4. D. Wang and T. Zhuang, Optics and lasers in engineering, 36, 3, (2001).

5. Roman Dolecek, Pavel Psota, Vít Lédl, Tomáš Vít, Jan Václavík, and Václav Kopecký, Applied Optics, 52, 1, (2013).

6. J. Smits, A. E. Perry, and P. H. Hoffmann, Journal of Physics E: Scientific Instruments, 11, 9, (2001) 